\title{
MÉTODO DA SUPERFÍCIE DE RESPOSTA APLICADO A VIGAS DE CONCRETO ARMADO REFORÇADAS COM CFRP
}

\author{
F. E. S. DA SILVA JÚNIOR \\ Mestre em Modelagem Computacional \\ Universidade Federal do Rio Grande \\ Rio Grande do Sul; Brasil \\ jrsuri@outlook.com
}

\author{
R. J. C. SILVA \\ Professor Doutor \\ Universidade Estadual Vale do Acaraú \\ Ceará; Brasil \\ ricardo.carvalho222@gmail.com
}

\author{
M. DE V. REAL \\ Professor Doutor \\ Universidade Federal do Rio Grande \\ Rio Grande do Sul; Brasil \\ mauroreal@furg.br
}

\section{RESUMO}

O Método dos Elementos Finitos é bastante consolidado na solução de problemas de engenharia. No entanto, em muitos casos o modelo pode exigir uma malha complexa e o número de simulações pode chegar à casa dos milhares, gerando um alto custo computacional. Para reduzir este custo existem algumas técnicas de otimização, como o Método da Superfície de Resposta, no qual é gerada uma função aproximadora capaz de descrever o sistema. O presente estudo buscou demonstrar a determinação da função de resposta de uma viga de concreto armado reforçada com polímeros reforçados com fibras de carbono (PRFC). Foi utilizado o software ANSYS para a modelagem em elementos finitos da viga, onde o modelo foi validado com dados experimentais, e foi utilizado o software Minitab para a obtenção da superfície de resposta. Através da análise das métricas do modelo, verificou-se boa adequação dos resultados.

Palavras-chave: modelagem computacional, método dos elementos finitos, método da superfície de resposta, otimização.

\section{ABSTRACT}

The Finite Element Method is well established in engineering problems solving. However, in many cases the model may require a complex mesh and the number of simulations may reach thousands, generating a high computational cost. To reduce this cost there are some optimization techniques, such as the Response Surface Methodology, which generates an approximation function capable of describing the system. The present study aimed to demonstrate the determination of the response function of a reinforced concrete beam strengthened with carbon fiber reinforced polymer (CFRP). The ANSYS software was used for the finite element modeling of the beam, where the model was validated with experimental data, and the Minitab software was used to obtain the response surface. Through the analysis of the model metrics, it was verified a good accuracy of the results.

Keywords: computational modeling; finite element method; response surface methodology; optimization.

\section{INTRODUÇÃO}

A análise computacional através do Método dos Elementos Finitos (MEF) tornou-se muito utilizada devido à fidelidade com a qual os modelos constitutivos de diversos materiais conseguem representar a realidade. No entanto, apesar de ser um método consolidado, dependendo das características da análise realizada, o MEF pode exigir uma malha complexa e se torna custoso computacionalmente. Se o método for utilizado em análises de confiabilidade com simulações de Monte Carlo, o tempo de processamento pode inviabilizar sua utilização.

Simulação de Monte Carlo é o processo repetitivo de geração de soluções de um problema com base na geração aleatória dos parâmetros de entrada. Segundo Ang e Tang (1984), como o método de Monte Carlo geralmente requer um número elevado de repetições, principalmente para problemas envolvendo eventos muito raros, sua aplicação pode se tornar custosa. Em diversos trabalhos, como em Wang et al. (2015), Jiang et al. (2015), Shi e Stewart (2015), Balomenos et al. (2018) e Roy (2019), recorreu-se à utilização de simulações de Monte Carlo associadas ao MEF. 
Para se reduzir o número de simulações de Monte Carlo existem algumas técnicas de otimização, como o Método da Superfície de Resposta (MSR). Para Silva e Rouboa (2014), combinar metodologias de otimização a modelos numéricos evita experimentos caros e demorados. Montgomery e Runger (2007) explicam que o MSR é uma coleção de técnicas matemáticas e estatísticas que são úteis para modelagem e análise de problemas nos quais uma resposta de interesse é influenciada por várias variáveis e o objetivo é otimizar essa resposta.

Fang e Tee (2017) destacam o alto custo computacional de métodos convencionais de análise de confiabilidade e demonstraram em seu trabalho a eficácia do MSR quando aplicado com este fim. Aliando-se esta técnica ao MEF, é realizado um pequeno número de simulações em elementos finitos e em seguida os resultados são aproximados através de uma função polinomial aproximadora. Assim, é possível abdicar o uso direto do MEF e realizar as análises de interesse apenas utilizando essa função.

Em Makki et al. (2018) foi desenvolvido um modelo em elementos finitos para prever o fator de concentração de tensão em epóxi reforçado com fibra de carbono, e em seguida os autores analisaram a confiabilidade deste fator utilizando os resultados do MSR. Em Kolios et al. (2018), um modelo em elementos finitos para conversores de energia de ondas foi desenvolvido e validado através de um modelo analítico, sendo aplicado o MSR para realizar análises de confiabilidade. A mesma abordagem foi utilizada em Gharaibeh (2018), onde foi analisada a confiabilidade de componentes eletrônicos submetidos a vibrações.

O presente estudo busca demonstrar a determinação da função de resposta de uma viga de concreto armado reforçada com polímeros reforçados com fibras de carbono (PRFC). Para isto, dividiu-se o trabalho em duas etapas: a primeira compreendeu a modelagem em elementos finitos no software ANSYS Workbench 17.1 de uma viga reforçada e a validação do modelo através de resultados experimentais; e a segunda etapa consistiu na obtenção da função aproximadora pelo Método da Superfície de Resposta pelo software Minitab 17 e pela análise das métricas do modelo polinomial.

\section{MODELAGEM COMPUTACIONAL}

\subsection{Modelos constitutivos}

Para o concreto, existem diferentes modelos aptos a representar o comportamento deste material. De acordo com Zhang et al. (2016), a abertura e propagação de fissuras ainda é um problema desafiador em estudos numéricos, já que a maioria dos modelos numéricos homogeneízam o efeito de fissuras em estruturas de concreto. O modelo constitutivo padrão que o software ANSYS utiliza para representar o concreto é o critério de Willam-Warnke (1974) (Figura 1) com cinco parâmetros para falha.

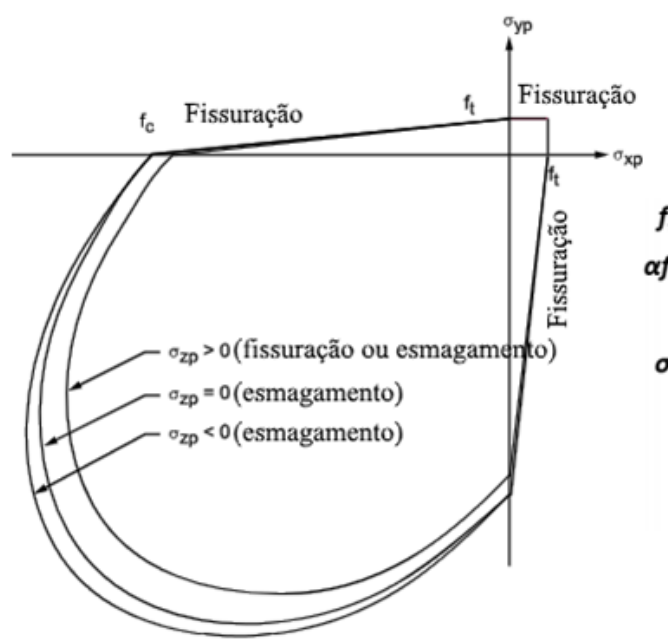

(a) Concreto comprimido

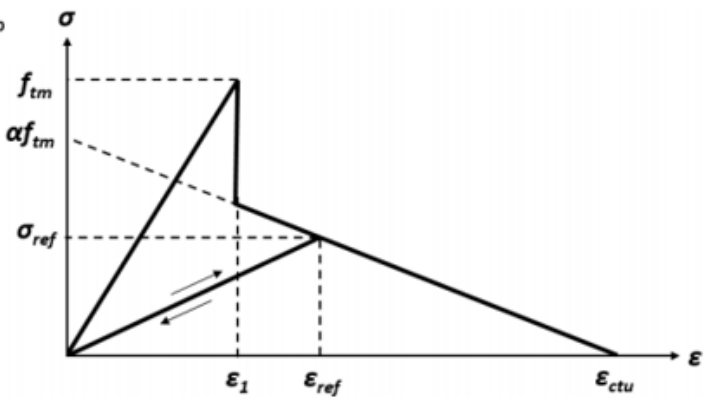

(b) Concreto tracionado

Figura 1: Critério de falha de Willam-Warnke. Fonte: adaptado de ANSYS (2013).

Para Lazzari et al. (2017), o modelo mais adotado para simulação do aço das armaduras em estruturas de concreto armado é o modelo uniaxial, onde considera-se que as armaduras resistem apenas a esforços axiais. Este modelo considera um comportamento elastoplástico representado por um diagrama tensão-deformação bilinear (Figura 2), e o material possui comportamento isotrópico. Para aços com patamar de escoamento bem definido, adota-se um modelo elastoplástico 
perfeito. Para os demais aços, adota-se um modelo com endurecimento linear. No presente trabalho optou-se por adotar o modelo elastoplástico perfeito.

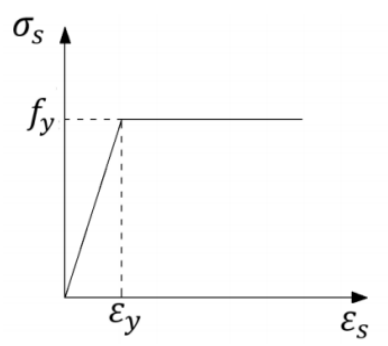

Figura 2: Modelo constitutivo do aço. Fonte: Barbosa (2017).

Apesar de boa parte das mantas de PRFC disponíveis no mercado possuírem orientação unidirecional, segundo Pivatto (2017), a consideração das propriedades ortotrópicas do material não influencia significativamente nos resultados em vigas. Desta forma, vale a Lei de Hooke para representação da fibra de carbono.

Mecânica da Fratura é a área da Mecânica dos Sólidos que estuda a propagação de fissuras em materiais. Como explica Anderson (2017), em um processo de abertura de fissuras, existem três modos de carregamento que proporcionam a propagação de uma fissura, conforme mostrado na Figura 3.

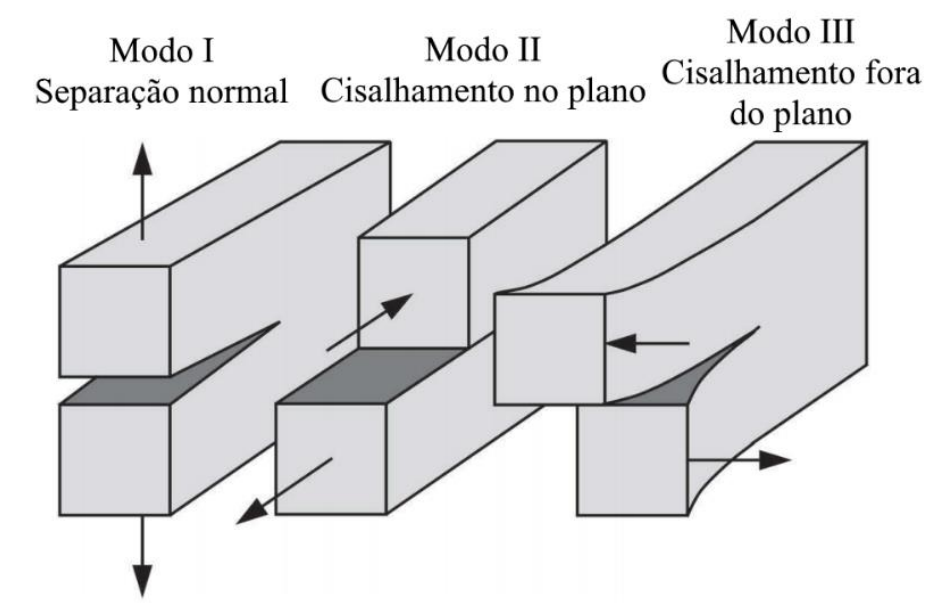

Figura 3: Modos de fratura. Fonte: adaptado de Anderson (2017).

Cohen et al. (2018) explicam que a interface concreto-PRFC é uma fina camada de adesivo onde a deformação relativa entre o PRFC e o concreto ocorre principalmente devido a esforços cortantes. Portanto, o modo de fratura é predominantemente o Modo II. A separação normal entre as faces dos materiais ainda existe, no entanto, geralmente é aceito que o descolamento da fibra corresponde somente ao Modo II, já que a camada de adesivo transfere esforços cortantes do concreto para o PRFC.

Um dos modelos de interface mais utilizados é o modelo bilinear de Lu et al. (2005), que utiliza os parâmetros de tensão de cisalhamento máxima na interface, deslizamento tangencial e energia de fratura. Kohnehpooshi e Jaafar (2017) destacam que esse é um dos modelos mais precisos para interface. De acordo com o modelo bilinear de Lu et al. (2005) (Figura 4), quando uma viga é carregada, uma tensão interfacial de cisalhamento $\tau$ surge devido à abertura de fissuras. Essa abertura é representada numericamente como um deslizamento finito entre a viga e o reforço. Inicialmente, quando o carregamento e a tensão interfacial $\tau$ são inferiores à capacidade resistente da interface $\tau_{\text {max }}$, a interface é considerada como estando no estágio linear-elástico. Esse estágio termina quando $\tau$ excede $\tau_{\max }$, o que significa que o sistema está na iminência de sofrer micro descolamentos. À medida em que o deslizamento continua, a tensão interfacial cai a zero e se inicia o descolamento completo do reforço, que se propaga ao longo da interface e representa o macro descolamento. 


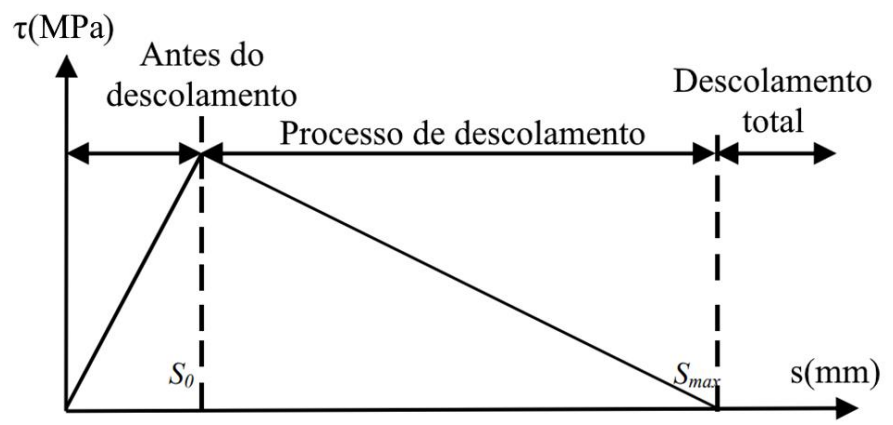

Figura 4: Modelo bilinear de Lu et al. (2005). Fonte: adaptado de Kohnehpooshi e Jaafar (2017).

\subsection{Modelo da viga estudada}

A armadura longitudinal da viga estudada foi dimensionada de acordo com a norma brasileira ABNT NBR 6118 (2014). Inicialmente, foi considerada uma viga de 5 metros de comprimento com seção retangular de $15 \mathrm{~cm}$ de base e $50 \mathrm{~cm}$ de altura, e submetida a um carregamento permanente de $13 \mathrm{kN} / \mathrm{m}$ e carregamento variável de $3 \mathrm{kN} / \mathrm{m}$. A viga foi considerada biapoiada nas duas extremidades. Para o dimensionamento do reforço, foram seguidas as diretrizes da norma americana ACI 440.2R (2017), sendo considerada uma situação onde a carga variável foi aumentada para $9 \mathrm{kN} / \mathrm{m}$. Desta forma, a viga foi dimensionada com duas barras de $16 \mathrm{~mm}$ para armadura longitudinal, 50 estribos de $6.3 \mathrm{~mm}$ com espaçamento de $10 \mathrm{~cm}$ e uma camada de reforço à flexão com PRFC, conforme detalhamento mostrado na Figura 5. Na Tabela 1 são apresentadas as propriedades dos materiais adotadas no dimensionamento.

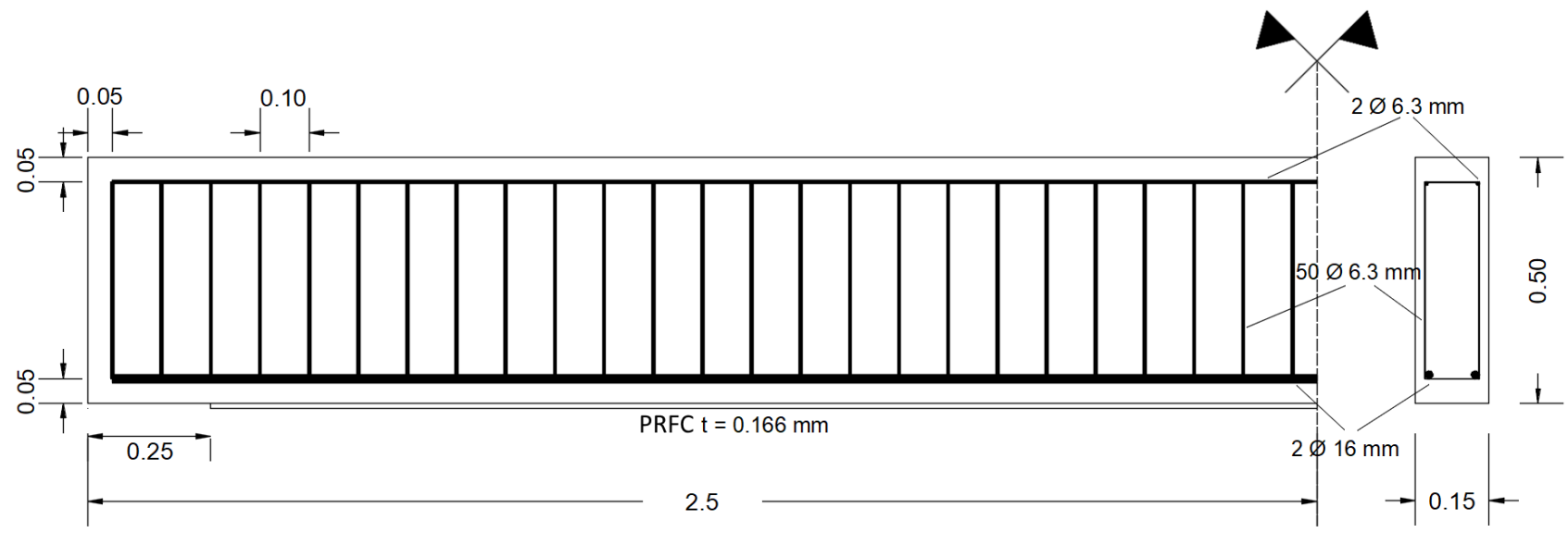

Figura 5: Modelo da viga estudada

Tabela 1 - Propriedades dos materiais (valores médios)

\begin{tabular}{c|c|c|c}
\hline Material & Parâmetro & Unidade & Valor \\
\hline \multirow{3}{*}{ Concreto } & $\mathrm{f}_{\mathrm{c}}$ & $\mathrm{MPa}$ & 30 \\
\cline { 2 - 4 } & $\mathrm{f}_{\mathrm{t}}$ & $\mathrm{MPa}$ & 2,9 \\
\cline { 2 - 4 } & $\mathrm{E}_{\mathrm{c}}$ & $\mathrm{GPa}$ & 500 \\
\hline \multirow{2}{*}{ Aço } & $\mathrm{f}_{\mathrm{y}}$ & $\mathrm{MPa}$ & 210 \\
\hline \multirow{3}{*}{ PRFC } & $\mathrm{E}_{\mathrm{s}}$ & $\mathrm{GPa}$ & 4900 \\
\cline { 2 - 4 } & $\mathrm{f}_{\mathrm{fu}}$ & $\mathrm{MPa}$ & 21 \\
\cline { 2 - 4 } & $\varepsilon_{\mathrm{fu}}$ & $\% \mathrm{or}$ & 230 \\
\cline { 2 - 4 } & $\mathrm{E}_{\mathrm{f}}$ & $\mathrm{GPa}$ & \\
\hline
\end{tabular}

\subsection{Procedimento de análise pelo MEF}

Nas simulações por elementos finitos, foram definidos os seguintes elementos: o elemento Solid65 para o concreto; o elemento de barra Link180 para as armaduras; o elemento Solid185 para o reforço e os pontos de carregamento e apoios; e os elementos Conta174 e Targe170 para as regiões de contato. 
Para o concreto, foram definidos ainda os coeficientes de fissura aberta, igual a 0,3 , e de fissura fechada, igual a 1 . Para o aço foi definido módulo tangente igual a 0 após o escoamento, ou seja, um modelo elastoplástico perfeito. Os coeficientes de Poisson adotados para o concreto, aço e reforço foram respectivamente 0,20, 0,30 e 0,27.

Para a região da interface, foi definido modo II de fratura. O programa oferece as opções de basear o modelo de interface na distância máxima de separação ou na energia de fratura. Foram realizados vários testes e foi observada melhor convergência da análise quando se utilizou o modelo baseado em energia de fratura. Para Anderson (2017), a abordagem da energia considera que a fratura ocorre quando a energia disponível para fissuração é suficiente para superar a resistência do material.

Todos os contatos foram definidos como bonded com a formulação pure penalty. Foi escolhido o contato bonded devido à maior facilidade de convergência por ser um tipo de contato linear, conforme explica Lee (2018), e as não-linearidades da região de contato foram introduzidas através da ferramenta Fracture do Workbench.

As análises utilizaram um critério de convergência baseado em deslocamento, onde foi definida uma tolerância de $1 \%$ e adotado o método de Newton-Raphson completo. O carregamento foi aplicado em um único passo de carga e 150 substeps (incrementos). O carregamento foi aplicado como força e as reações de apoio foram definidas como deslocamentos nulos nos eixos X e Y nos pontos de apoio. O parâmetro observado na resposta da estrutura ao carregamento foi sua carga de ruína, caracterizada pela interrupção das simulações com uma mensagem de erro de convergência indicando carregamento excessivo.

\subsection{Método da Superfície de Resposta}

O Método da Superfície de Resposta busca representar, através de uma função aproximadora baseada nos resultados de um pequeno número de simulações de Monte Carlo, a influência de cada uma das variáveis da função de desempenho. A função aproximadora pode ser linear ou quadrática, e é aproximada através da regressão dos resultados discretos obtidos numericamente. A forma genérica para uma superfície de resposta é apresentada na Equação (1), onde y é a função da superfície de resposta genérica, $a_{0}, a_{i}$ e $a_{i j}$ são coeficientes ajustados via regressão dos dados, e $x$ são variáveis aleatórias.

$y=a_{0}+\sum_{i=1}^{N} a_{i} x_{i}+\sum_{i=1}^{N} \sum_{j=1}^{N} a_{i j} x_{i} x_{j}$

Análise de regressão é um método estatístico que utiliza a relação entre duas ou mais variáveis independentes para que uma variável dependente possa ser estimada a partir delas. Ou seja, a regressão determina a relação entre as variáveis de entrada e de saída. Existem alguns tipos de regressão, como a regressão linear múltipla, onde os dados são aproximados para uma função linear e existe mais de uma variável independente, e a regressão polinomial, onde os dados são aproximados para uma função polinomial, com a condição de que deve haver apenas uma variável independente. No caso de várias variáveis independentes e da necessidade de uma função polinomial, é utilizado o Método da Superfície de Resposta.

Para a viga em estudo, foram definidos os seguintes parâmetros de entrada (variáveis independentes): resistência à compressão do concreto $\left(f_{c}\right)$, tensão de escoamento do aço $\left(f_{y}\right)$, altura útil da viga $(d)$ e módulo de elasticidade do reforço $\left(E_{f}\right)$, e a variável de saída (dependente) foi a carga de ruptura $\left(P_{u}\right)$.

Foi adotada a técnica de amostragem Central Composite Design para a geração dos design points (pontos de projeto), que foram gerados automaticamente pelo ANSYS utilizando sua ferramenta Response Surface. Os resultados de cada design point foram inseridos no software Minitab para obtenção da superfície de resposta.

\section{VALIDAÇÃO DO MODELO EM ELEMENTOS FINITOS}

Para validação do modelo, foram modeladas vigas de três trabalhos distintos, buscando representar diversas situações de reforço: as vigas de Pivatto (2017), de Ferreira et al. (2018) e de Beber (2003). Devido à simetria das peças analisadas, foi modelado apenas um quarto das vigas para redução do esforço computacional e foi aplicada simetria nos eixos X e Z. O modelo adotado está ilustrado na Figura 6.

As vigas de Pivatto (2017) apresentavam $220 \mathrm{~cm}$ de comprimento, $25 \mathrm{~cm}$ de altura e $15 \mathrm{~cm}$ de largura, e a armadura longitudinal de tração era composta por duas barras de $12,5 \mathrm{~mm}$. Uma viga (V1) recebeu uma camada de reforço e sem ancoragem; outra viga (V2) recebeu duas camadas de reforço e nenhuma ancoragem; a terceira viga (VA1) recebeu uma camada de reforço e um par de ancoragens laterais; e a quarta viga (VAA1) recebeu uma camada de reforço e dois pares de ancoragens laterais.

As vigas de Ferreira et al. (2018) apresentavam $140 \mathrm{~cm}$ de comprimento, $20 \mathrm{~cm}$ de altura e $12 \mathrm{~cm}$ de largura, e a armadura longitudinal de tração era composta por duas barras de $8 \mathrm{~mm}$. Uma viga (V2) recebeu uma camada de reforço com $57 \mathrm{~cm}$ de comprimento e um par de ancoragens laterais, e as ancoragens apresentavam $10 \mathrm{~cm}$ de largura; a viga V3 recebeu uma camada de reforço com $77 \mathrm{~cm}$ de comprimento e um par de ancoragens laterais, e as ancoragens apresentavam $10 \mathrm{~cm}$ de 


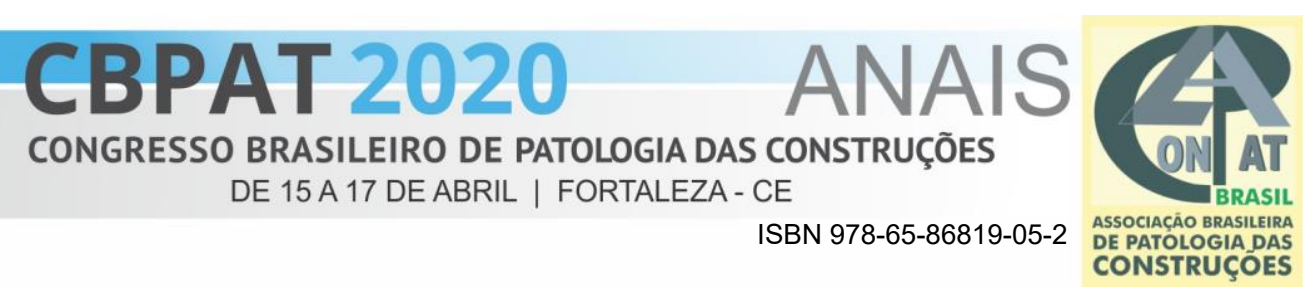

largura; e a viga V4 recebeu uma camada de reforço com $77 \mathrm{~cm}$ de comprimento e um par de ancoragens laterais com 15 $\mathrm{cm}$ de largura.
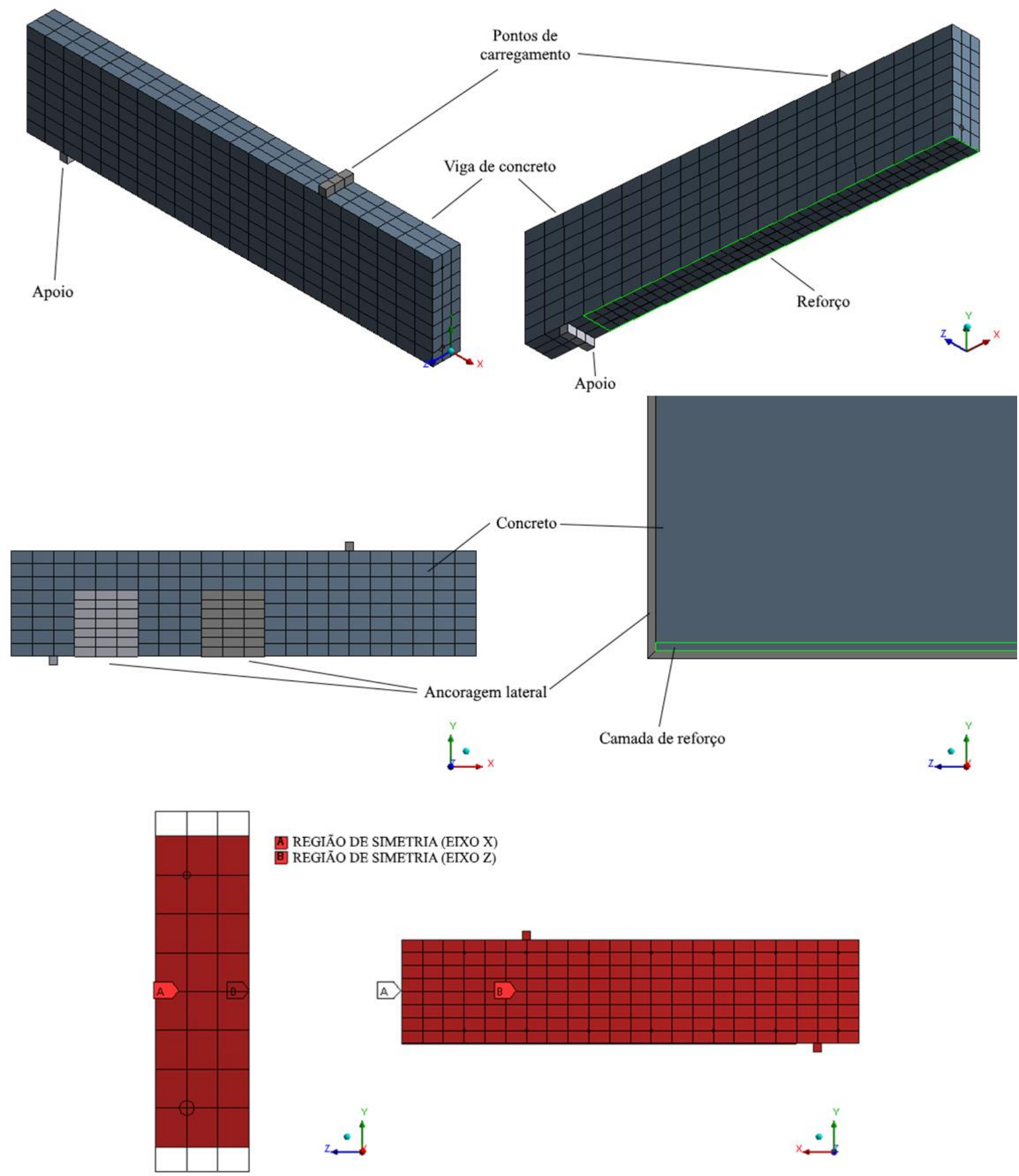

Figura 6: Modelo de elementos finitos para as vigas

As vigas de Beber (2003) apresentavam $300 \mathrm{~cm}$ de comprimento, $30 \mathrm{~cm}$ de altura e $15 \mathrm{~cm}$ de largura, e a armadura longitudinal de tração era composta por duas barras de 12,5 mm. Uma viga (V6) recebeu uma camada de reforço com $238 \mathrm{~cm}$ de comprimento sem ancoragem lateral, e o reforço apresentava 9,5 cm de largura; a outra viga (V7) recebeu 
quatro camadas de reforço com $238 \mathrm{~cm}$ de comprimento sem ancoragem lateral, e o reforço apresentava a mesma largura da viga.

A malha de todas as vigas foi concebida com $5 \mathrm{~cm}$ na direção $X$. No eixo $Y$, a malha foi dividida em oito elementos de dimensões iguais nesta direção, e no eixo $\mathrm{Z}$, a malha foi dividida em três elementos de dimensões iguais nesta direção. Os pontos de carregamento e apoios foram modelados com $2 \mathrm{~cm}$ de largura.

Nas vigas com mais de uma camada de reforço, todas as camadas foram modeladas juntas como um único sólido para simplificação do modelo e redução da quantidade de contatos. A malha dos reforços foi refinada na direção X, ficando com $25 \mathrm{~mm}$ nesta direção, pois o programa recomenda que seja refinada a malha nessa região.

Todas as vigas foram ensaiadas através do ensaio de flexão de quatro pontos. Mais detalhes encontram-se em Pivatto (2017), Ferreira et al. (2018) e Beber (2003). As propriedades dos materiais são apresentadas na Tabela 3.

Tabela 2 - Propriedades dos materiais para as vigas da validação do modelo

\begin{tabular}{c|c|c|c|c|c}
\hline \multirow{3}{*}{ Material } & Parâmetro & Unidade & $\begin{array}{c}\text { Pivatto } \\
\mathbf{( 2 0 1 7 )}\end{array}$ & $\begin{array}{c}\text { Ferreira } \\
\text { et al. (2018) }\end{array}$ & Beber (2003) \\
\hline \multirow{3}{*}{ Concreto } & $\mathrm{f}_{\mathrm{c}}$ & $\mathrm{MPa}$ & 24,05 & 20 & 32,8 \\
\cline { 2 - 6 } & $\mathrm{f}_{\mathrm{t}}$ & $\mathrm{MPa}$ & 2,2 & 2,21 & 2,9 \\
\cline { 2 - 6 } & $\mathrm{E}_{\mathrm{c}}$ & $\mathrm{GPa}$ & 25,714 & 25,044 & 26 \\
\hline \multirow{3}{*}{ Aço } & $\mathrm{f}_{\mathrm{y}}$ & $\mathrm{MPa}$ & 550 & 500 & 706,5 \\
\cline { 2 - 6 } & $\mathrm{E}_{\mathrm{s}}$ & $\mathrm{GPa}$ & 210 & 210 & 210 \\
\hline \multirow{3}{*}{ PRFC } & $\mathrm{t}$ & $\mathrm{mm}$ & 0,176 & 0,166 & 0,176 \\
\cline { 2 - 6 } & $\mathrm{f}_{\mathrm{fu}}$ & $\mathrm{MPa}$ & 3800 & 4900 & 3800 \\
\cline { 2 - 6 } & $\varepsilon_{\mathrm{fu}}$ & $\%$ & 17 & 21 & 240 \\
\cline { 2 - 6 } & $\mathrm{E}_{\mathrm{f}}$ & $\mathrm{GPa}$ & 240 & 230 & 240 \\
\hline
\end{tabular}

Os resultados da validação do modelo em elementos finitos são apresentados na Tabela 3.

Tabela 3 - Resultados da validação do modelo

\begin{tabular}{|c|c|c|c|c|}
\hline \multicolumn{2}{|c|}{ Viga } & $\mathbf{P}_{\mathrm{u}, \mathrm{exp}} *$ & $\mathbf{P}_{\mathrm{u}, \mathrm{MEF}}{ }^{* *}$ & $\begin{array}{c}\text { Variação } \\
(\%)\end{array}$ \\
\hline \multirow{4}{*}{$\begin{array}{c}\text { Pivatto } \\
(2017)\end{array}$} & V1 & 112,67 & 113,72 & 0,93 \\
\hline & V2 & 114,41 & 118,42 & 3,50 \\
\hline & VA1 & 117,74 & 118,01 & 0,23 \\
\hline & VAA1 & 120,85 & 120,53 & $-0,26$ \\
\hline \multirow{3}{*}{$\begin{array}{l}\text { Ferreira } \\
\text { et al. }(2018)\end{array}$} & V2 & 66,00 & 62,68 & $-5,03$ \\
\hline & V3 & 66,00 & 66,38 & 0,58 \\
\hline & V4 & 73,00 & 68,40 & $-6,30$ \\
\hline \multirow{2}{*}{$\begin{array}{l}\text { Beber } \\
(2003)\end{array}$} & V6 & 118,53 & 118,80 & 0,23 \\
\hline & V7 & 160,93 & 151,60 & $-5,80$ \\
\hline
\end{tabular}

$* \mathrm{P}_{\mathrm{u}, \mathrm{exp}}$ : Carga de ruptura experimental;

$* * \mathrm{P}_{\mathrm{u}, \mathrm{MEF}}$ : Carga de ruptura através do modelo em elementos finitos.

Constatou-se uma boa aproximação entre cargas de ruptura experimentais e numéricas. Observou-se que em mais da metade dos resultados a diferença foi menor que $1 \%$. Ocorreram ainda resultados cuja variação foi da ordem de $5 \%$, no entanto, em análises de confiabilidade, geralmente é considerada a variável de erro do modelo para cobrir as incertezas provenientes do modelo utilizado. Desta forma, considerou-se o modelo em elementos finitos adequado para previsão de carga de ruptura e prosseguiu-se para a modelagem da viga do presente estudo.

\section{RESULTADOS E DISCUSSÕES}

A viga em estudo foi modelada conforme o exposto nos itens 2 e 3 e na Figura 6. Devido à variação na altura útil da viga entre os pontos de projeto, a malha de elementos foi refinada na direção $\mathrm{Y}$, apresentando 40 elementos nesta direção com $12,5 \mathrm{~mm}$ cada, pois o elemento Solid65 exige que a armadura seja posicionada nas arestas dos elementos de concreto, necessitando de um grande refinamento da malha nesta direção para que houvesse essa coincidência em todos os design points. Ainda assim, pela técnica Central Composite Design, foram gerados design points com alturas úteis de $41,11 \mathrm{~cm}$ e 46,39 cm. Para evitar um refinamento ainda maior da malha, foi utilizado o comando CEINTF, o qual estabelece uma 
tolerância para "puxar" a armadura para as arestas dos elementos de concreto durante o processo de análise. Assim, na prática, as alturas úteis consideradas nesses design points foram de 41,25 cm e 46,25 cm (ver Tabela 4).

Foram realizadas um total de 29 simulações com a viga modelada. Em todas as situações, o modo de ruptura observado foi o desprendimento do reforço com escoamento da armadura de tração e esmagamento do concreto comprimido, e não ocorreu ruptura da fibra.

Os pontos de projeto, cargas de ruptura de cada simulação e a diferença entre o valor observado e os valores previstos pelo modelo polinomial são apresentados na Tabela 4. Os coeficientes da função polinomial de resposta e os coeficientes de determinação (S e $\mathrm{R}^{2}$ ) retornados pelo Minitab são mostrados na Figura 7.

Tabela 4 - Pontos de projeto e resultados dos dois modelos

\begin{tabular}{|c|c|c|c|c|c|c|c|c|}
\hline d $(\mathbf{c m})$ & $\mathbf{f}_{\mathrm{c}}(\mathbf{M P a})$ & $\mathbf{f}_{\mathbf{y}}(\mathbf{M P a})$ & $\mathbf{E}_{\mathbf{f}}(\mathbf{G P a})$ & $\begin{array}{c}\mathbf{P}_{\mathbf{u}, \mathbf{M E F}} \\
(\mathbf{k N})^{*}\end{array}$ & $\begin{array}{c}\mathbf{P}_{\mathbf{u}, \mathbf{S R}} \\
(\mathbf{k N}) * *\end{array}$ & $\begin{array}{c}\mathbf{P}_{\mathrm{u}, \mathrm{SR} /} \\
\mathbf{P}_{\mathrm{u}, \mathrm{MEF}} \\
(\%)\end{array}$ & Resíduo & $\begin{array}{l}\text { Resíduo } \\
\text { padron. }\end{array}$ \\
\hline 40,00 & 30,00 & 500,00 & 230,00 & 180,58 & 181,56 & 0,54 & $-2,46$ & $-1,41$ \\
\hline 41,25 & 26,48 & 464,79 & 215,92 & 179,48 & 177,31 & $-1,22$ & 0,67 & 0,44 \\
\hline 41,25 & 26,48 & 464,79 & 244,08 & 179,38 & 178,45 & $-0,52$ & $-0,55$ & $-0,37$ \\
\hline 41,25 & 26,48 & 535,21 & 244,08 & 199,58 & 197,30 & $-1,15$ & 0,70 & 0,53 \\
\hline 41,25 & 33,52 & 464,79 & 215,92 & 197,38 & 194,56 & $-1,45$ & 1,35 & 1,11 \\
\hline 41,25 & 33,52 & 464,79 & 244,08 & 201,08 & 197,66 & $-1,73$ & 1,96 & 1,38 \\
\hline 41,25 & 33,52 & 535,21 & 215,92 & 201,28 & 200,48 & $-0,40$ & $-0,77$ & $-0,51$ \\
\hline 41,25 & 33,52 & 535,21 & 244,08 & 207,98 & 206,28 & $-0,82$ & 0,14 & 0,08 \\
\hline 43,75 & 25,00 & 500,00 & 230,00 & 198,38 & 194,94 & $-1,76$ & 1,79 & 1,07 \\
\hline 43,75 & 30,00 & 450,00 & 230,00 & 197,58 & 198,37 & 0,40 & $-2,37$ & $-1,45$ \\
\hline 43,75 & 30,00 & 500,00 & 210,00 & 203,88 & 201,55 & $-1,16$ & 0,69 & 0,41 \\
\hline 43,75 & 30,00 & 500,00 & 230,00 & 209,58 & 204,19 & $-2,64$ & 3,74 & 1,87 \\
\hline 43,75 & 30,00 & 500,00 & 250,00 & 209,78 & 209,14 & $-0,30$ & $-1,00$ & $-0,59$ \\
\hline 43,75 & 30,00 & 550,00 & 230,00 & 218,28 & 215,43 & $-1,32$ & 1,14 & 0,68 \\
\hline 43,75 & 35,00 & 500,00 & 230,00 & 216,58 & 217,87 & 0,59 & $-2,92$ & $-1,75$ \\
\hline 46,25 & 26,48 & 464,79 & 215,92 & 198,78 & 196,51 & $-1,15$ & 0,54 & 0,29 \\
\hline 46,25 & 26,48 & 464,79 & 244,08 & 203,38 & 201,41 & $-0,98$ & 0,25 & 0,16 \\
\hline 46,25 & 26,48 & 535,21 & 215,92 & 211,78 & 211,92 & $\mathbf{0 , 0 7}$ & $-1,96$ & $-1,34$ \\
\hline 46,25 & 33,52 & 464,79 & 215,92 & 220,58 & 219,83 & $-0,34$ & $-0,96$ & $-0,66$ \\
\hline 46,25 & 33,52 & 464,79 & 244,08 & 229,38 & 226,69 & $-1,18$ & 0,99 & 0,90 \\
\hline 46,25 & 33,52 & 535,21 & 215,92 & 227,18 & 225,00 & $-0,96$ & 0,37 & 0,20 \\
\hline 46,25 & 33,52 & 535,21 & 244,08 & 233,68 & 234,57 & 0,38 & $-2,69$ & $-1,47$ \\
\hline 47,50 & 30,00 & 500,00 & 230,00 & 221,58 & 217,18 & $-2,03$ & 2,58 & 1,56 \\
\hline 45,00 & 30,00 & 500,00 & 230,00 & 209,78 & 209,59 & $-0,09$ & $-1,52$ & $-0,74$ \\
\hline 45,00 & 25,00 & 450,00 & 210,00 & 188,48 & 187,45 & $-0,55$ & $-0,62$ & $-0,42$ \\
\hline 45,00 & 30,00 & 550,00 & 210,00 & 216,98 & 216,02 & $-0,44$ & $-0,82$ & $-0,48$ \\
\hline 45,00 & 35,00 & 550,00 & 210,00 & 227,68 & 224,63 & $-1,36$ & 1,29 & 0,85 \\
\hline 45,00 & 25,00 & 500,00 & 250,00 & 204,68 & 203,89 & $-0,39$ & $-0,92$ & $-0,63$ \\
\hline 45,00 & 35,00 & 550,00 & 250,00 & 241,38 & 238,26 & $-1,31$ & 1,36 & 0,91 \\
\hline
\end{tabular}

*P $\mathrm{P}_{\mathrm{u}, \mathrm{MEF}}$ : Carga de ruptura através do modelo em elementos finitos;

$* * \mathrm{P}_{\mathrm{u}, \mathrm{SR}}$ : Carga de ruptura através do modelo polinomial

Para que o modelo seja considerado adequado, segundo a documentação do Minitab, algumas condições devem ser atendidas:

a) No gráfico de resíduos x ajustes (Figura 8), não deve ocorrer um espalhamento desigual de resíduos entre os valores previstos, não deve se formar um padrão curvilíneo, não deve haver um ponto muito distante de zero nem muito distante dos outros na direção X;

b) No gráfico de resíduos x ordem (Figura 9), não deve se formar um padrão ou tendência;

c) No gráfico de probabilidade normal dos resíduos (Figura 10), os pontos devem seguir aproximadamente uma linha reta. 


\section{СВРAT 2020 \\ CONGRESSO BRASILEIRO DE PATOLOGIA DAS CONSTRUÇÕES \\ DE 15 A 17 DE ABRIL | FORTALEZA - CE \\ ISBN 978-65-86819-05-2

Model Summary

$\begin{array}{rrrr}\text { S } & \text { R-sq } & \text { R-sq(adj) } & \text { R-sq(pred) } \\ 2,32095 & 98,92 \% & 97,84 \% & 95,50 \%\end{array}$

Coded Coefficients

\begin{tabular}{|c|c|c|c|c|c|c|}
\hline Term & Effect & Coef & SE Coef & T-Value & P-Value & VIF \\
\hline Constant & & 205,83 & 1,17 & 175,94 & 0,000 & \\
\hline . & 35,967 & 17,983 & 0,839 & 21,43 & 0,000 & 1,14 \\
\hline fc & 22,905 & 11,453 & 0,717 & 15,97 & 0,000 & 1,23 \\
\hline y & 17,198 & 8,599 & 0,734 & 11,72 & 0,000 & 1,29 \\
\hline & 7,585 & 3,793 & 0,684 & 5,54 & 0,000 & 1,21 \\
\hline $\mathrm{d}$ & $-9,64$ & $-4,82$ & 1,67 & $-2,89$ & 0,012 & 1,09 \\
\hline$={ }^{*} \mathrm{fc}$ & 4,42 & 2,21 & 1,41 & 1,57 & 0,139 & 1,35 \\
\hline *fy & 5,42 & 2,71 & 1,45 & 1,86 & 0,083 & 1,44 \\
\hline$=* E f$ & 2,30 & 1,15 & 1,38 & 0,83 & 0,419 & 1,33 \\
\hline fc & 6,47 & 3,23 & 1,37 & 2,36 & 0,033 & 1,23 \\
\hline fy & $-0,79$ & $-0,40$ & 1,39 & $-0,29$ & 0,780 & 1,26 \\
\hline Ef & 4,01 & 2,00 & 1,33 & 1,51 & 0,153 & 1,17 \\
\hline$z^{*} f_{y}$ & $-10,32$ & $-5,16$ & 1,16 & $-4,46$ & 0,001 & 1,47 \\
\hline$={ }^{*} \mathrm{E}$ & 1,98 & 0,99 & 1,07 & 0,93 & 0,370 & 1,58 \\
\hline$f^{\star} E f$ & 2,73 & 1,36 & 1,10 & 1,24 & 0,235 & 1,67 \\
\hline
\end{tabular}

Regression Equation in Uncoded Units

$P u=9+24,5 d-2,52$ fc $-0,514$ fy $-3,28$ Ef $-0,343 d^{*} d+0,0884$ fc*fc $+0,001083$ fy* fy

$+0,00288$ Ef*Ef $+0,1725 d^{\star} f c-0,00211 d^{\star} f y+0,0267 d^{\star} E f-0,02063$ f $c^{\star} f_{Y}$

$+0,0099$ fckEf $+0,00136$ fy*Ef

Figura 7: Parâmetros do modelo polinomial

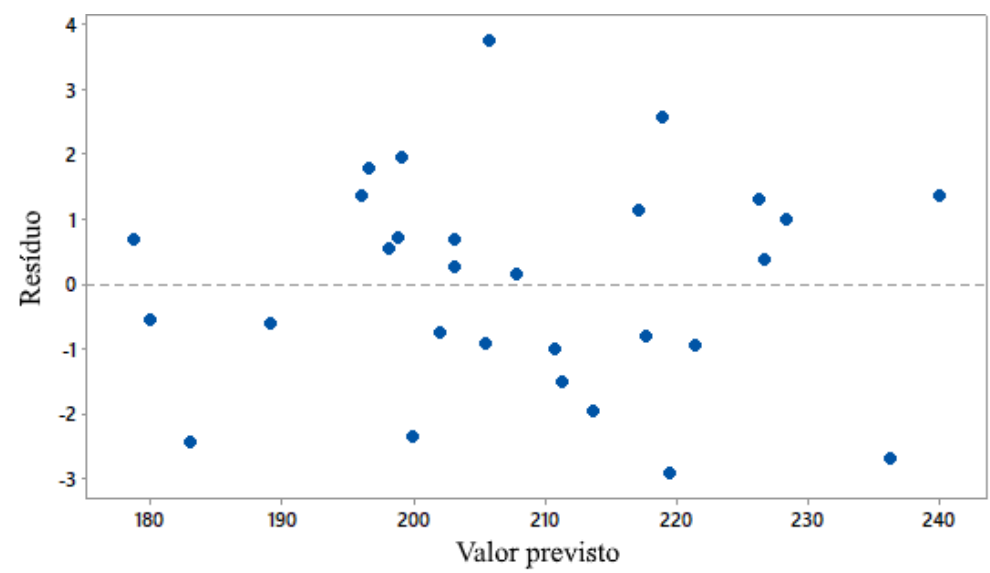

Figura 8: Resíduos x ajustes

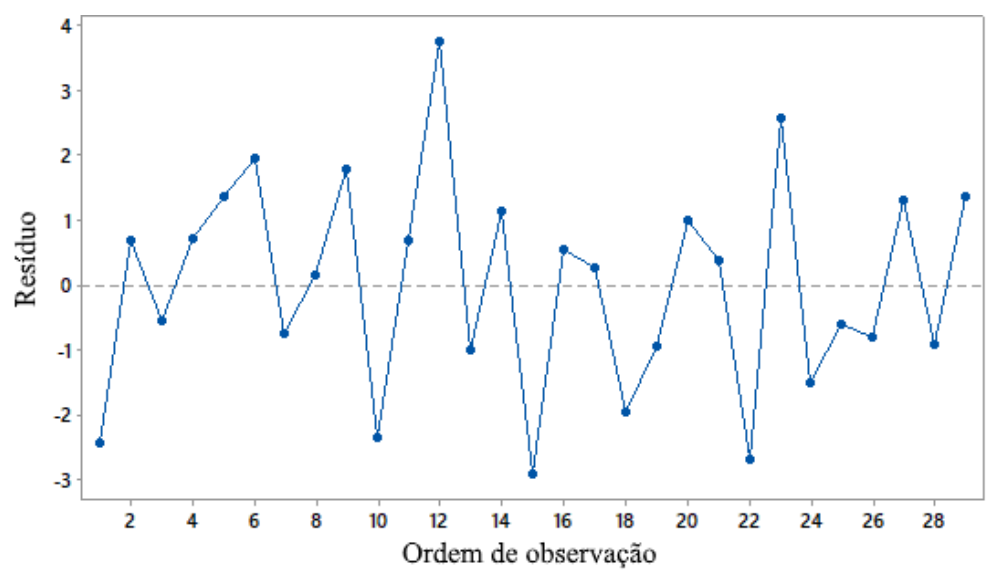

Figura 9: Resíduos x ordem 


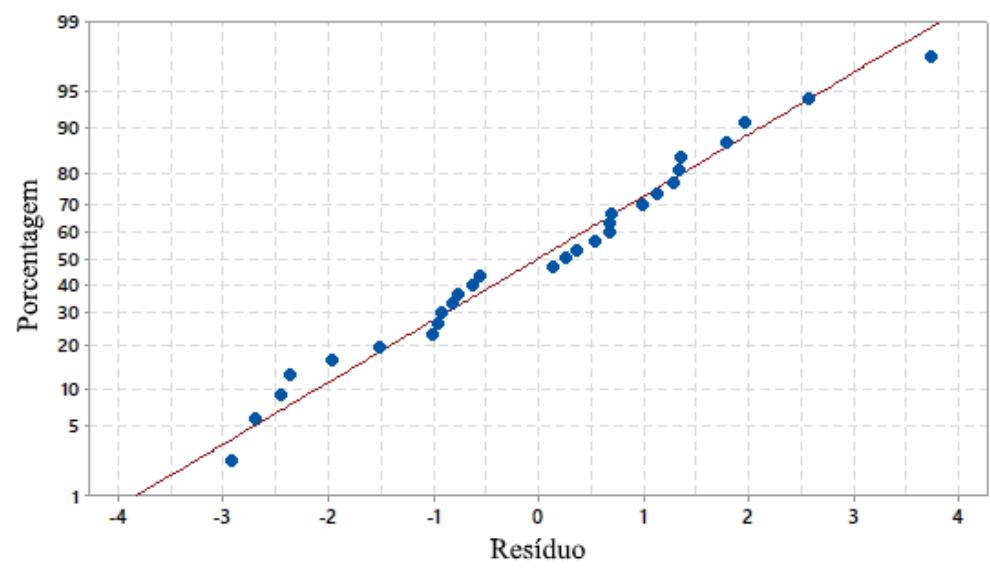

Figura 10: Probabilidade normal dos resíduos

Como observado na Figura 7 e Tabela 5, os valores previstos pelo modelo foram próximos aos valores observados, e obteve-se resíduos sempre inferiores a 2. O modelo apresentou um $R^{2}$ e $R^{2}$ ajustado próximos a 1 , o que significa que aproximadamente $98 \%$ da variação na carga de ruptura é explicada pela variação dos dados de entrada. Além disso, o valor de $S$ (raiz quadrada do erro quadrado médio) foi baixo quando comparado aos valores previstos de $P_{u}$. De acordo com a documentação do Minitab, $S$ é medido nas unidades da variável de resposta e representa a variação de quão longe os valores dos dados estão da superfície de resposta verdadeira. Quanto menor o valor de $S$, melhor o modelo descreve a resposta.

Observando-se os gráficos das Figuras 8, 9 e 10, verifica-se que as condições para um bom ajuste foram atendidas. Portanto, pode-se dizer que a superfície de resposta encontrada se ajustou bem aos dados e pode ser utilizada para prever a carga de ruptura da viga em estudo.

Por fim, foi observada a influência de cada parâmetro de entrada na carga de ruptura da viga. Verificou-se que o valor da resistência $P_{u}$ é diretamente proporcional a todos os parâmetros de entrada. Essas observações podem ser verificadas na Figura 11, enquanto na Figura 12 são apresentados os gráficos das interações entre as variáveis.

Pela análise dos gráficos das Figuras 11 e 12, as variáveis de maior impacto na carga de ruptura foram a resistência à compressão do concreto e a tensão de escoamento do aço. O módulo de elasticidade da fibra, embora também influencie na carga última, não possui tanta importância como as demais variáveis. Além disso, a altura útil parece ter sua influência reduzida à medida em que $d$ é aumentado além de $45 \mathrm{~cm}$. Observou-se ainda a influência das combinações entre variáveis e foi observado que a combinação de $f_{c}$ e $f_{y}$ é a mais determinante na resistência da viga.

Verificou-se um bom ajuste do modelo aos dados observados em cada design point. As variáveis de entrada do modelo foram capazes de explicar cerca de $98 \%$ da variação na carga de ruptura. A função obtida foi capaz de representar adequadamente a variação na carga de ruptura da viga, observando-se uma diferença máxima de $2 \%$ entre resultados pelo MEF e pelo modelo polinomial.

Desta forma, a superfície obtida pode ser utilizada para outras finalidades. Tomando como exemplo uma análise de confiabilidade, onde o número de simulações de Monte Carlo chegaria facilmente aos milhares devido à baixa probabilidade de falha da estrutura, conseguiu-se reduzir este número para apenas 29 simulações pelo MEF, o que demonstra a capacidade deste método de otimização.

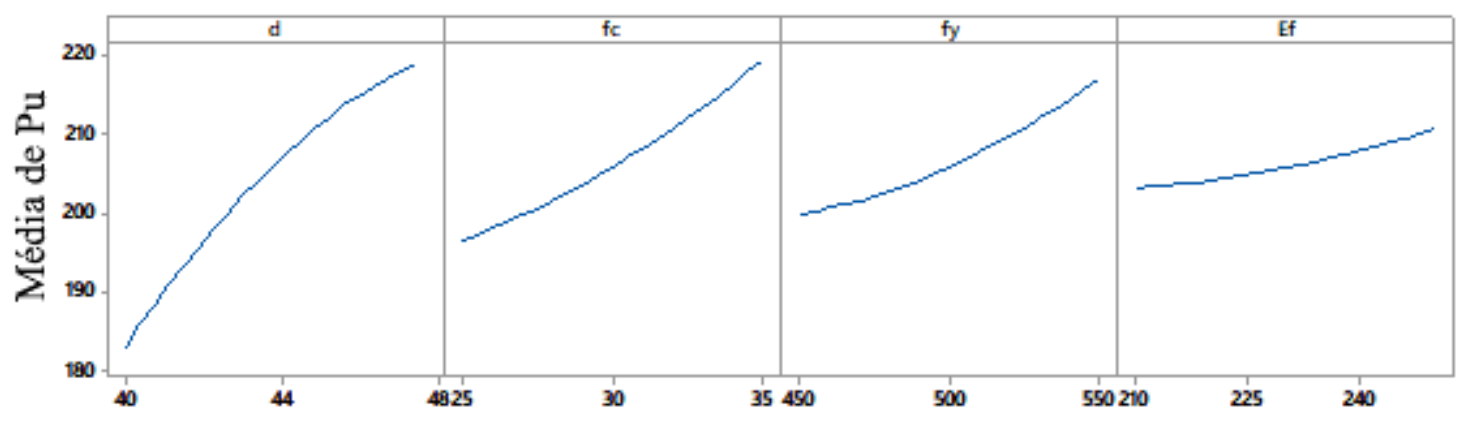

Figura 11: Influência das variáveis 


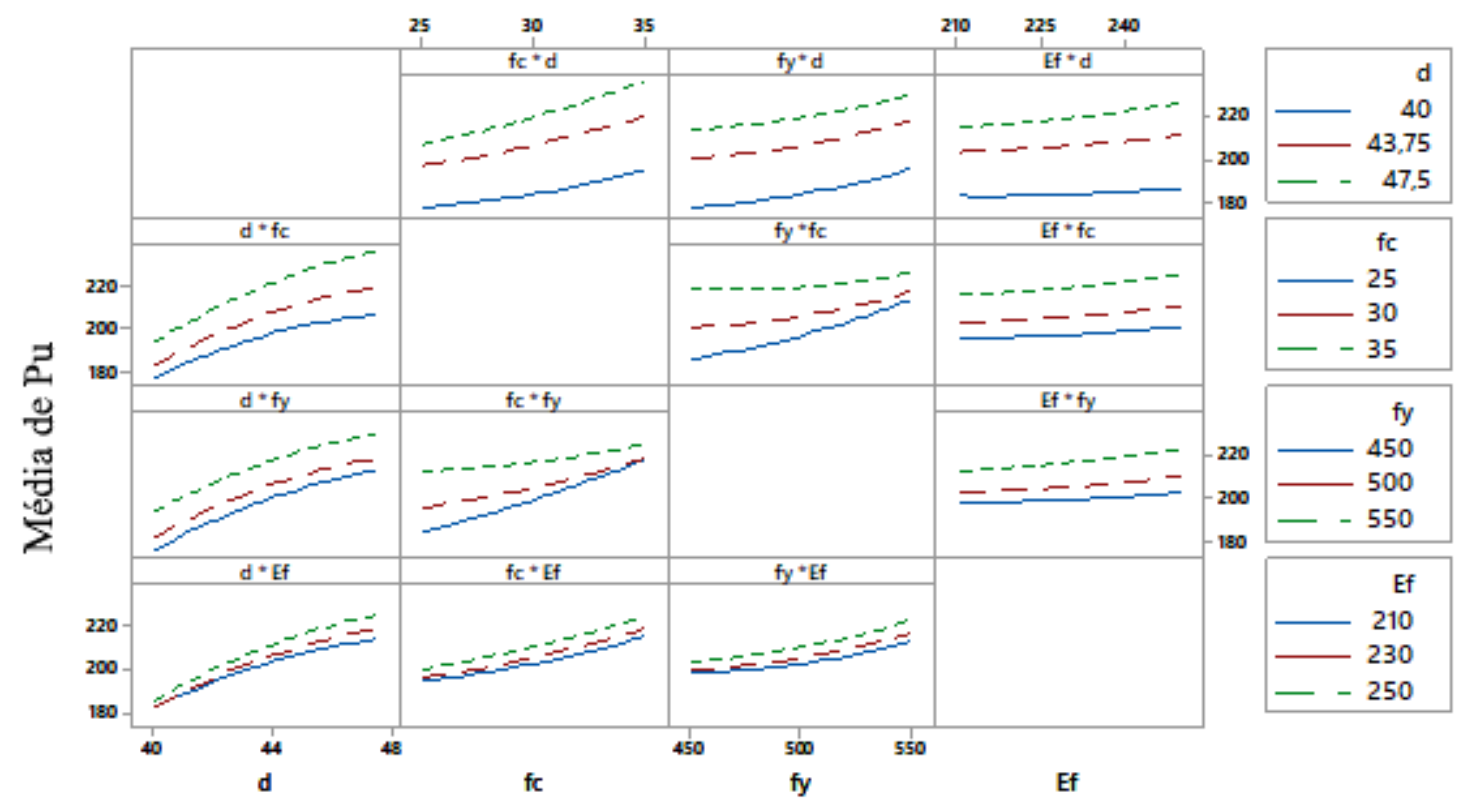

Figura 12: Interação entre as variáveis

\section{CONCLUSÕES}

Neste estudo foi realizada a modelagem em elementos finitos de uma viga de concreto armado reforçada com PRFC seguida da aplicação do Método da Superfície de Resposta. Por fim, foi obtida a função aproximadora da carga de ruptura do sistema. Algumas conclusões puderam ser tiradas.

a) $\mathrm{O}$ modelo em elementos finitos foi validado com dados experimentais e mostrou adequado funcionamento. Considerar o efeito da interface entre concreto-PRFC foi de fundamental importância, uma vez que o modo de ruptura da viga analisada foi o descolamento do reforço.

b) O modelo polinomial mostrou boa aproximação com os dados observados em cada design point. A função obtida foi capaz de representar adequadamente a variação na carga de ruptura da viga, podendo ser utilizada para diversos fins e reduzindo drasticamente o esforço computacional que seria empregado no caso de uma grande quantidade de simulações pelo MEF.

c) Foram verificadas as métricas da superfície de resposta encontrada e observou-se um bom ajuste, sendo que as variáveis de entrada do modelo foram capazes de explicar cerca de $98 \%$ da variação na carga de ruptura.

d) As variáveis de maior impacto na carga de ruptura foram a resistência à compressão do concreto e a tensão de escoamento do aço. O módulo de elasticidade da fibra, embora também influencie na carga última, não possui tanta importância como as demais variáveis.

Por fim, espera-se que a metodologia apresentada no presente estudo possa contribuir para futuras pesquisas sobre o tema, principalmente em análises de confiabilidade.

\section{REFERÊNCIAS}

AMERICAN CONCRETE INSTITUTE. ACI 440.2R: Guide for the Design and Construction of Externally Bonded FRP Systems for Strengthening Concrete Structures. Farmington Hills, 2017.

ANDERSON, T. L. Fracture mechanics: fundamentals and applications. Boca Ratón: CRC Press, 2017.

ANG, A. H. S.; TANG, W. H. Probability concepts in engineering planning and design, vol. 2: decision, risk, and reliability. New York: John Wiley \& Sons, 1984.

ANSYS INC. ANSYS Mechanical APDL Material Reference [Manual], Release 15.0. Canonsburg, 2013.

ASSOCIAÇÃO BRASILEIRA DE NORMAS TÉCNICAS. NBR 6118: Projeto de estruturas de concreto Procedimento. Rio de Janeiro, 2014. 
BALOMENOS, G. P.; GENIKOMSOU, A. S.; POLAK, M. A. Investigation of the effect of openings of interior reinforced concrete flat slabs. Structural Concrete, v. 6, n. 19, p. 1672-1681, 2018.

BARBOSA, P. R. O. Análise probabilística de pilares de concreto armado através do método dos elementos finitos. 2017. Dissertação de Mestrado - Universidade Federal do Rio Grande do Sul - UFRGS, Porto Alegre, RS, Brasil, 2017.

BEBER, A. J. Comportamento estrutural de vigas de concreto armado reforçadas com compósitos de fibra de carbono. 2003. Tese de Doutorado - Universidade Federal do Rio Grande do Sul - UFRGS, Porto Alegre, RS, Brasil, 2003.

COHEN, M.; MONTELEONE, A.; POTAPENKO, S. Finite Element Analysis of Intermediate Crack Debonding in FRP Strengthened RC Beams. Canadian Journal of Civil Engineering, v. 45, n. 10, p. 840-851, 2018.

FANG, Y.; TEE, K. F. Structural reliability analysis using response surface method with improved genetic algorithm. Structural Engineering and Mechanics, v. 62, n. 2, p. 139-142, 2017.

FERREIRA, M. P.; OLIVEIRA, M. H.; LIMA NETO, A. F.; TAPAJÓS, L. S. Influência da ancoragem na resistência à flexão de vigas reforçadas com mantas de PRFC. Revista Alconpat, v. 9, n. 1, p. 30-47, 2019.

GHARAIBEH, M. A. Reliability analysis of vibrating electronic assemblies using analytical solutions and response surface methodology. Microelectronics Reliability, v. 84, p. 238-247, 2018.

JIANG, Y.; SUN, G.; HE, Y.; BEER, M.; ZHANG, J. A nonlinear model of failure function for reliability analysis of RC frame columns with tension failure. Engineering Structures, v. 98, p. 74-80, 2015.

KOHNEHPOOSHI, O. M. I. D.; JAAFAR, M. S. Non-linear three dimensional finite elements for composite concrete structures. Latin American Journal of Solids and Structures, v. 14, n. 3, p. 398-421, 2017.

KOLIOS, A.; DI MAIO, L. F.; WANG, L.; CUI, L.; SHENG, Q. Reliability assessment of point-absorber wave energy converters. Ocean Engineering, v. 163, p. 40-50, 2018.

LAZZARI, B. M.; CAMPOS FILHO, A.; LAZZARI, P. M.; PACHECO, A. R. Using element-embedded rebar model in ANSYS for the study of reinforced and prestressed concrete structures. Computers and Concrete, v. 19, n. 4, p. 347-356, 2017.

LEE, H. Finite Element Simulations with ANSYS Workbench 18. Mission: SDC publications, 2018.

LU, X. Z.; TENG, J. G.; YE, L. P.; JIANG, J. J. Bond-slip models for FRP sheets/plates bonded to concrete. Engineering Structures, v. 27, n. 6, p. 920-937, 2005.

MAKKI, M. M.; AHMED, B.; CHOKRI, B. Reliability prediction of the stress concentration factor using response surface method. The International Journal of Advanced Manufacturing Technology, v. 94, n. 1-4, p. 817-826, 2018.

MOntGomery, D. C.; RUNGer, G. C. Applied Statistics And Probability For Engineers. Hoboken: John Wiley \& Sons, 2007.

PIVATTO, A. B. Análise experimental e computacional de vigas biapoiadas de concreto armado reforçadas com CRFC. 2017. Dissertação de Mestrado - Universidade Tecnológica Federal do Paraná - UTFPR, Curitiba, PR, Brasil, 2017.

ROY, A. Efficient reliability analysis of concrete structures on the basis of non-linear finite element analysis under numerical noise. Tese de Doutorado - Delft University of Technology, Delft, Países Baixos, 2019.

SHI, Y.; STEWART, M. G. Spatial reliability analysis of explosive blast load damage to reinforced concrete columns. Structural Safety, v. 53, p. 13-25, 2015. 


\section{СВРAT 2020 \\ CONGRESSO BRASILEIRO DE PATOLOGIA DAS CONSTRUÇÕES \\ DE 15 A 17 DE ABRIL | FORTALEZA - CE \\ ISBN 978-65-86819-05-2}

SILVA, V.; ROUBOA, A. Optimizing the gasification operating conditions of forest residues by coupling a twostage equilibrium model with a response surface methodology. Fuel Processing Technology, v. 122, p. 163-169, 2014.

WANG, X.; YANG, Z.; JIVKOV, A. P. Monte Carlo simulations of mesoscale fracture of concrete with random aggregates and pores: a size effect study. Construction and Building Materials, v. 80, p. 262-272, 2015.

WILlAM, K. J.; WARNKE, E. D. Constitutive Model for the Triaxial Behavior of Concrete. Proceedings, International Association for Bridge and Structural Engineering, v. 19, p. 174, 1974.

ZHANG, D.; WANG, Q.; DONG, J. Simulation study on CFRP strengthened reinforced concrete beam under fourpoint bending. Computers and Concrete, v. 17, n. 3, p. 407-421 2016. 